\title{
PROPERTIES OF SWELLING AND SHRINKING OF POLY- (ACRYLAMIDE-CO-ACRYLIC ACID) GELS BY INFINITESIMAL CHANGES IN EXTERNAL CONDITIONS
}

\author{
NAKANO YOSHIO, NARUOKA HIROHITO \\ AND MURASE MATSUHIKO \\ Department of Chemical Engineering, Faculty of Engineering, \\ Shizuoka University, Hamamatsu 432
}

Key Words : Copolymer Gel, Infinitesimal Change, Swelling, Equilibrium, Kinetics

\begin{abstract}
The phase transition of a gel was investigated from the viewpoint of both equilibrium and kinetics. Poly(acrylamide-co-acrylic acid) gels synthesized in our laboratory showed drastic changes in volume by infinitesimal change in concentration of acetone-water mixtures. Such phenomena took place near a critical concentration of the solvent. The equilibrium degree of phase transition of the gel was analysed, using the mean field theory proposed by Flory-Huggins. It was found that the volume change was mainly represented by the $f$-value defined in the Flory-Huggins formula. The diffusivity of the gel network was estimated from the concentration swing experiment near the phase transition and the equation of motion of gel network. The diffusivities, $D$, were correlated well with the $f$-values; as $D=\exp (0.486 f-13.9)$ for the synthesized copolymer gels.
\end{abstract}

\section{Introduction}

Gels are important substances employed widely in the fields of biochemistry and bioengineering. The physical chemistry of various gels has been studied intensively since early contributions of notable importance were made by Flory. ${ }^{2)}$

Recently, Tanaka et al. ${ }^{6-8)}$ have discovered unsuspected phenomena in gels, namely drastic volume changes in a polymer gel which can be brought about by changes in external conditions such as temperature, ${ }^{4,6)} \mathrm{pH},{ }^{6,7)}$ solvent composition, ${ }^{3,5,6)}$ ionic composition $^{6,7)}$ or electric field. ${ }^{8)}$ These phenomena have been interpreted as a phase transition in gels, and the phase equilibrium of gels mainly has been studied theoretically. However, only a little work has been done on the kinetics of volume change in a gel when it approaches equilibrium. From a practical point of view, this might provide important information on large-scale fluctuations in pore size ${ }^{1)}$ and other properties. Furthermore, no one has yet reported studies on the influence of the polymer network structure, represented by the relative amount of main-chain molecules, cross-linked molecules and ionizable molecules, on the kinetics of volume change as well as the phase transition in an ionic gel.

The objective of the investigation described in this paper is to show quantitatively the relationships

Received November $9, \overline{1985}$. Correspondence concerning this article should be addressed to $Y$. Nakano. among the polymer constituents (acrylamide, $N, N^{\prime}$ methylenebisacrylamide and acrylic acid), the equilibrium of the phase transition and the kinetics of swelling of poly(acrylamide-co-acrylic acid) gels. Poly(acrylamide-co-acrylic acid) gels were synthesized in our laboratory and acetone-water mixtures were employed as a solvent in this study. The volume change of the polymer by infinitesimal change in concentration of the solvent was measured and quantitatively explained according to the Flory-Huggins theory ${ }^{2)}$ for the swollen gel. It is shown that the diffusion coefficient of the gel network can be correlated with a factor correlated to the change in gel volume for gels of various constituents.

\section{Sample Preparation and Experimental Procedures}

Poly(acrylamide-co-acrylic acid) gels were synthesized in our laboratory from monomers. A typical procedure is as follows: Acrylamide (main component) and $N, N^{\prime}$-methylene-bisacrylamide (crosslinking agent) were used as received. Acrylic acid (ionizable component) was purified by reducedpressure distillation to remove an inhibitor. These monomers were dissolved in distilled, degassed and nitrogen-saturated water at $303 \mathrm{~K}$ to a final volume of $50 \mathrm{ml}$ (Solution A). Solution B of an accelerator, tetramethyl ethylene diamine (TEMED: $1.16 \mathrm{~g}$ ), and solution $\mathrm{C}$ of an initiator, ammonium persulfate $(1.71 \mathrm{~g})$, were prepared in a similar manner as for solution $\mathrm{A}$. Then, known quantities of each solution 
were mixed fully and transferred to small glass tubes $(0.692,1.05,1.54,2.18 \mathrm{~mm}$ i.d. $)$, where polymerization took place for five minutes at $303 \mathrm{~K}$. The gels were left in the tubes for an hour. Then they were removed and cut into rod-shaped pieces, having a length about equal to the diameter. The gels were immersed in a large amount of pure water for two days to wash away unreacted substances.

In equilibrium experiments, the gel was immersed in acetone-water mixtures having various compositions until it reached equilibrium state. The final diameter of the cross section of the rod-shaped gel was measured under a microscope.

In concentration swing experiments, the gel was put into a solution with somewhat higher than critical concentration of acetone, in which the state of the gel became highly sensitive. Then it was transferred to another solution with lower acetone concentration. The time course of the change in diameter of gel was measured in the same way as in the equilibrium experiments. The same procedure was applied to other gels.

\section{Results and Discussion}

The polymer compositions were varied as shown in Table 1 to investigate their effect on both the phase transition and the kinetics of swelling of gels.

3.1 Effect of acrylic acid fraction (charge density) in polymer

Figure 1 shows the degree of swelling of gels made using constant concentrations of acrylamide, Am, bisacrylamide, B, and various amounts of acrylic acid, Ac, as shown in Table 1. The abscissa in Fig. 1 shows the ratio of the volume fraction of the network, $\phi / \phi_{0}$; $\phi_{0}$ at the preparation and $\phi$ at equilibrium. The equilibrium volume of the gel, $V$ can be represented as follows:

$$
\frac{V_{0}}{V}=\frac{\phi}{\phi_{0}}
$$

where $V_{0}$ is the volume of the gel at $\phi=\phi_{0}$.

The gel which did not contain acrylic acid showed a continuous volume change, while gels having a large amount of Ac showed discontinuous volume transitions. In general, gels remained swollen when they were put into a solution with a low concentration of acetone. When the fraction of acetone became greater, the gels shrunk. Such a phase transition took place drastically near the critical concentration of acetone-water mixtures for each gel.

3.2 Effect of acrylamide fraction (polymerization density) in polymer

Figure 2 shows the degree of swelling of gels having fixed concentrations of $\mathrm{Ac}$ and $\mathrm{B}$, and various amounts of Am, as shown in Table 1. When the amount of Am in gels decreased, discontinuous vol-
Table 1. Compositions of poly(acrylamide-co-acrylic acid) gels.

\begin{tabular}{|c|c|c|c|}
\hline Key & $\underset{\left[\mathrm{mol}^{\prime} \mathrm{m}^{-3}\right]}{A c^{x}}$ & $\underset{\left[\mathrm{mol}^{\prime} \cdot \mathrm{m}^{-3}\right]}{\mathrm{A} \mathrm{m}^{\mathbf{x x}}}$ & $\begin{array}{c}\mathrm{B}^{\mathrm{xxx}} \\
{\left[\mathrm{mol} \cdot \mathrm{m}^{-3}\right]}\end{array}$ \\
\hline 0 & 20.42 & 689.4 & 9.081 \\
\hline (1) & 0.00 & \multirow{5}{*}{689.4} & \multirow{5}{*}{9.081} \\
\hline 0 & 10.21 & & \\
\hline$\theta$ & 61.26 & & \\
\hline D & 122.5 & & \\
\hline & 204.2 & & \\
\hline$\square$ & \multirow{3}{*}{20.42} & 590.9 & \multirow{3}{*}{9.081} \\
\hline $\bar{\square}$ & & 984.8 & \\
\hline & & 1379 & \\
\hline$\triangle$ & \multirow{3}{*}{20.42} & \multirow{3}{*}{689.4} & 6.811 \\
\hline$\Delta$ & & & 13.62 \\
\hline & & & 22.70 \\
\hline \multicolumn{4}{|c|}{ TEMED conc. $20.08\left[\mathrm{~mol} \cdot \mathrm{m}^{-3}\right]$} \\
\hline \multicolumn{4}{|c|}{$\begin{aligned} x & : \text { Acrylic Acid } \\
x \times & : \text { Acrylamide } \\
x \times x & : \text { Bisacrylamide }\end{aligned}$} \\
\hline
\end{tabular}

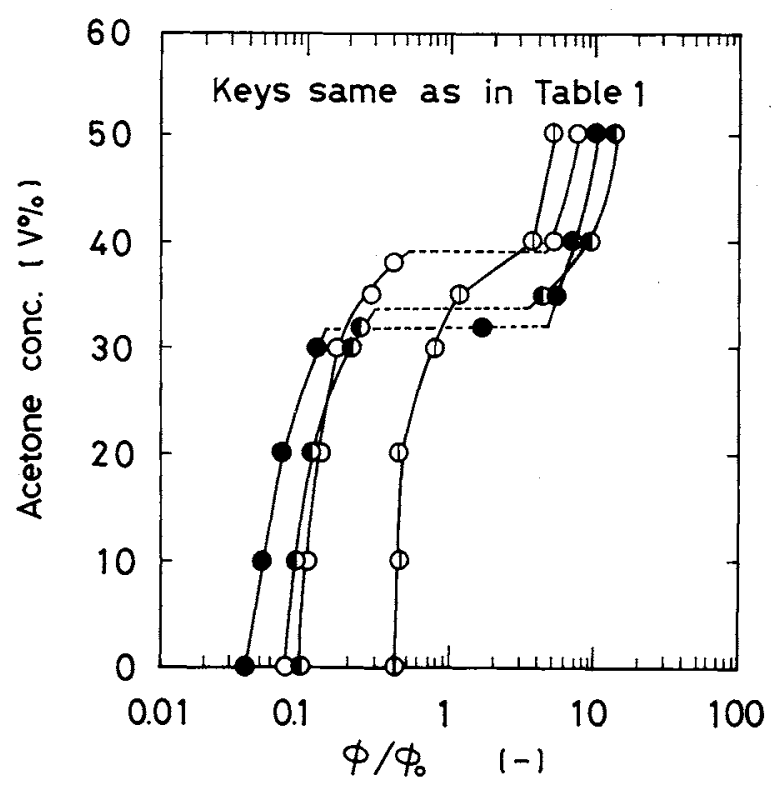

Fig. 1. Equilibrium degree of swelling of poly(acrylamide$c o$-acrylic acid) gels when only the concentrations of acrylic acid, Ac, are varied.

ume transitions were observed near the critical concentration. Gels having a large amount of Am, however, changed their volumes continuously. 


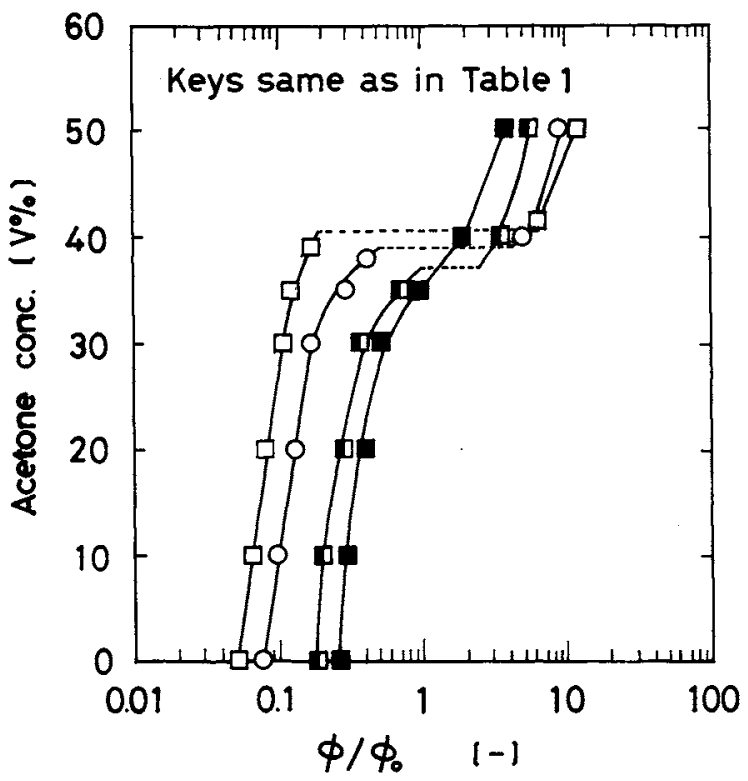

Fig. 2. Equilibrium degree of swelling of poly(acrylamideco-acrylic acid) gels when only the concentrations of acrylamide, Am, are varied.

\subsection{Effect of $N, N^{\prime}$-methylenebisacrylamide fraction (cross-linking density) in polymer}

Figure 3 shows the degree of swelling of gels having fixed concentrations of $\mathrm{Ac}$ and $\mathrm{Am}$, and various amounts of $\mathrm{B}$, as shown in Table 1. The volume changes of gels having a small amount of $\mathrm{B}$ were remarkably discontinuous, while gels having larger concentrations of B showed smaller volume changes.

\subsection{Effects of network structure on phase transition of gels}

The network structures of gels in this study are represented by the relative amounts of three components, as shown in Table 1. The phase transitions in gels are brought about by three forces: such as the polymer-polymer affinity, the rubber elasticity and the hydrogen-ion pressure. These forces constitute totally the osmotic pressure of the gel, because they determine whether the gel tends to take up solvent or to expel it. In the mean field theory the osmotic pressure of an ionic gel can be described by the FloryHuggins formula. ${ }^{2)}$

$$
\begin{aligned}
\Pi= & -\frac{N k T}{v}\left[\phi+\ln (1-\phi)+\frac{\Delta F}{2 k T} \phi^{2}\right] \\
& +v k T\left[\frac{\phi}{2 \phi_{0}}-\left(\frac{\phi}{\phi_{0}}\right)^{1 / 3}\right]+v f k T\left(\frac{\phi}{\phi_{0}}\right)
\end{aligned}
$$

The osmotic pressure of a gel must be zero for the gel to be in equilibrium with the surrounding solvent. From Eq. (2), this condition can be expressed as follows:

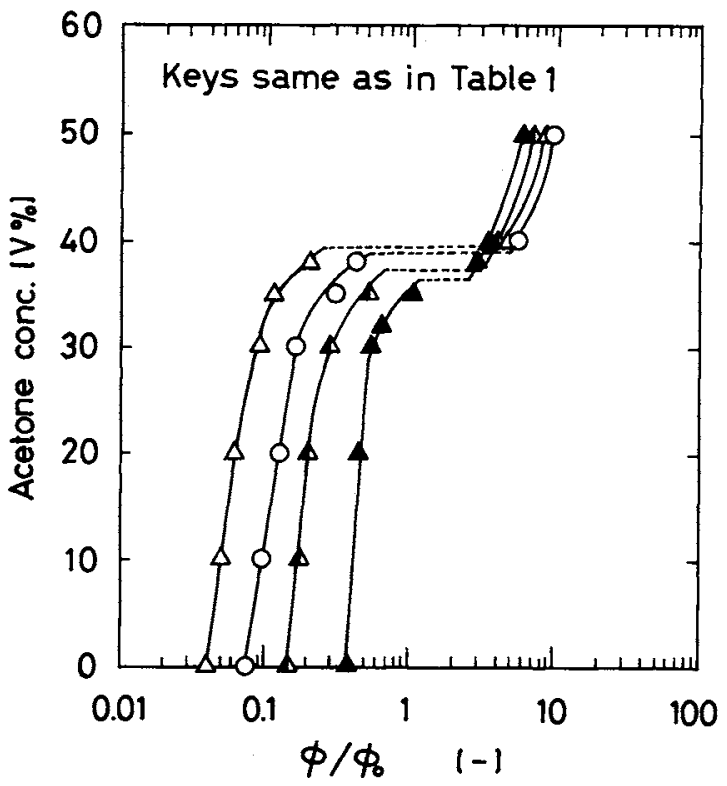

Fig. 3. Equilibrium degree of swelling of poly(acrylamideco-acrylic acid) gels when only the concentrations of bisacrylamide, $B$, are varied.

$$
\begin{aligned}
\tau=1 & -\frac{\Delta F}{k T}=-\frac{v v}{N \phi_{2}}\left[(2 f+1)\left(\frac{\phi}{\phi_{0}}\right)-2\left(\frac{\phi}{\phi_{0}}\right)^{1 / 3}\right] \\
& +1+\frac{2}{\phi}+\frac{2 \ln (1-\phi)}{\phi^{2}}
\end{aligned}
$$

where $N$ is Avogadro's number; $k$ is the Boltzmann constant; $T$ is the absolute temperature; $v$ is the molar volume of the solvent; $\Delta F$ is the free-energy decrease associated with the formation of contact between polymer segments; $v$ is the number of constituent chains per unit volume at $\phi=\phi_{0}$ and $f$ is the number of dissociated hydrogen ions per effective polymer chain.

The left-hand side of Eq. (3) may be called the. reduced temperature, $\tau$, which changes with temperature and solvent composition. The reduced temperature, $\tau$, can be determined as a function of the network concentration of gels. Among the parameters $\phi_{0}, \phi, v, v$ and $f$ on the right-hand side of Eq. (3), the values of $\phi_{0}, \phi$ and $v$ can be determined experimentally and the value of $v$ may be calculated from the amount of tetramethyl ethylene-diamine (TEMED). Therefore, the value of $f$ can be estimated by fitting the width of the phase transitions plotted on Figs. 1-3 with Eq. (3).

The value of $f$ in Eq. (3) is defined as the number of dissociated hydrogen ions per effective polymer chain. Therefore, it can also be estimated according to the following arguments. The probability for a crosslinking molecule to be incorporated simultaneously in two polymer chains $(\mathrm{Ac}+\mathrm{Am})$ can be estimated by considering a "reaction volume", $V_{\text {reac }}$, which defines a volume around a cross-linking molecule. When the 
polymer end having a free radical passes through the volume, this probability is given by $[(\mathrm{Am}+$ Ac) $\left.V_{\text {reac }}\right]^{2} .^{9)}$ The total number of ionizable molecules, Ac, per liter is $\mathrm{Ac} \cdot N$ and the number of crosslinking molecules, $\mathrm{B}$, per liter is $\mathrm{B} \cdot N$. In the polymer making up the cross-linked networks, the number of effective polymer chains is given by $\left(\frac{1}{2} \mathrm{~B}\right)[(\mathrm{Am}+$ Ac) $\left.V_{\text {reac }}\right]^{2} \cdot N$. Thus the value of $f$ can be given as follows.

$$
f=\frac{2 \mathrm{Ac}}{B\left[(\mathrm{Ac}+\mathrm{Am}) V_{\text {reac }}\right]^{2}}
$$

The above equation means that the volume transition becomes larger for gels containing a higher concentration of Ac; a lower concentration of Am and/or B.

Figure 4 shows the relationship between the $f$ values and $2 \mathrm{Ac} /\left[\mathrm{B}(\mathrm{Am}+\mathrm{Ac})^{2}\right]$. All the results are on a straight line except those for gels having Ac larger than $61.26 \mathrm{~mol} / \mathrm{m}^{3}$ at the fixed $\mathrm{Am}\left(689.4 \mathrm{~mol} / \mathrm{m}^{3}\right)$ and B $\left(9.081 \mathrm{~mol} / \mathrm{m}^{3}\right)$. In these gels the high concentration of acrylic acid might reduce the ionization due to charge repulsion, resulting in a smaller $f$-value. In addition, the value of $V_{\text {reac }}$ was obtained as $2.041 / \mathrm{mol}$ from the slope of the straight line.

\subsection{Volume changes in gels by infinitesimal changes in} concentration of acetone and water mixtures

To investigate the kinetics of swelling of gels, the swing in concentrations was performed near the critical concentration of acetone where a drastic volume change in the gel took place. Gels shrunken in a solvent with higher concentration of acetone were moved quickly into another solvent with a lower one. As an example, the time course of the swelling and shrinking of gels having the compositions of Ac $\left(20.42 \mathrm{~mol} / \mathrm{m}^{3}\right), \mathrm{Am}\left(689.4 \mathrm{~mol} / \mathrm{m}^{3}\right)$ and B $(9.081$ $\mathrm{mol} / \mathrm{m}^{3}$ ) are shown in Fig. 5. From the experimental results, it was found that the swelling-shrinking cycle was fully reversible. Tanaka et al. ${ }^{10)}$ recently proposed an equation of motion of a gel network. This equation has been applied only to the analysis of swelling of a nonionic gel, in which the degree of swelling is small. In the present study, this procedure was extended to the analysis of an ionic gel (acrylamide-acrylic acid copolymer gel) which showed drastic volume changes.

The kinetics of the swelling of a spherical gel can be described by the knowledge of a displacement vector, $\boldsymbol{u}(\boldsymbol{r}, t)$, as a function of space and time. The magnitude of displacement can be given by the following equation, based on the concept of Newton's second law and Hooke's law.

$$
\frac{\partial u}{\partial t}=D \frac{\partial}{\partial r}\left\{\frac{1}{r^{2}}\left[\frac{\partial}{\partial r}\left(r^{2} u\right)\right]\right\}
$$

with boundary and initial conditions as follows:

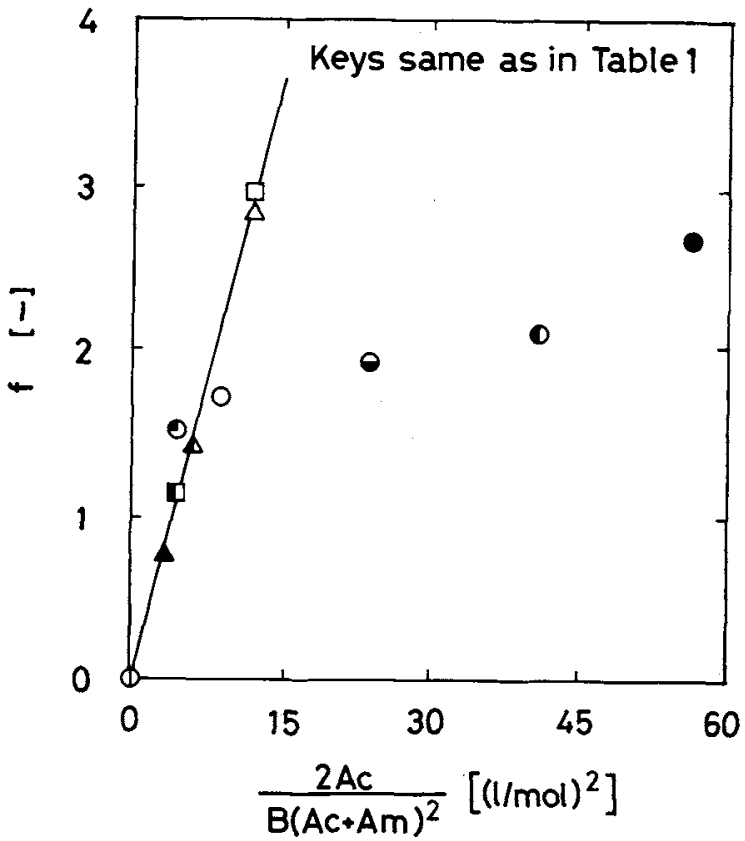

Fig. 4. Relationship between $f$-value of poly(acrylamideco-acrylic acid) gels determined from swelling experiments and $2 \mathrm{Ac} /\left[\mathrm{B}(\mathrm{Ac}+\mathrm{Am})^{2}\right]$ determined by preparation.

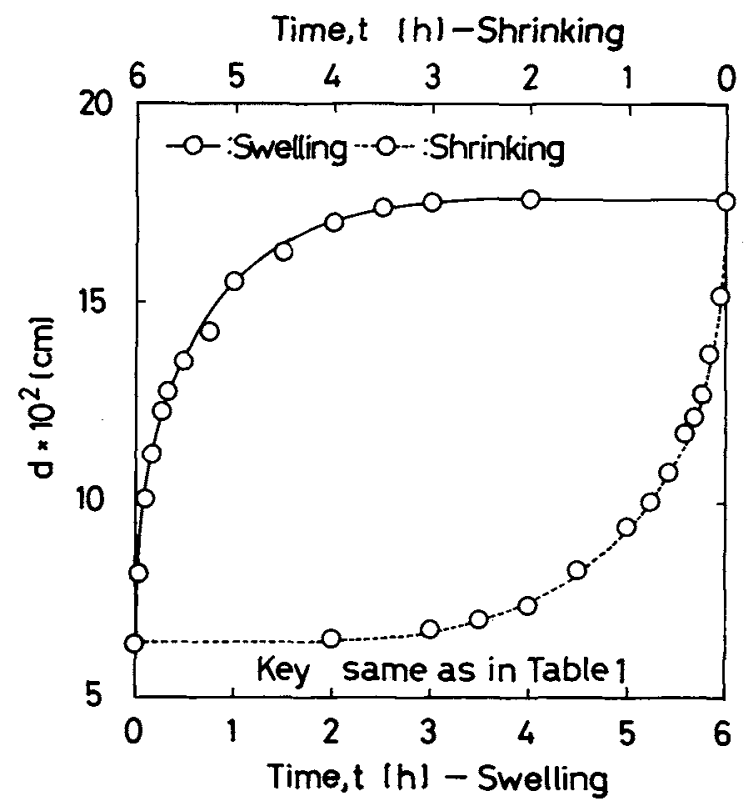

Fig. 5. Time courses of swelling and shrinking of gels having standard composition ( $O$ key in Table 1).

$$
\begin{aligned}
& \text { B.C } \sigma_{r r}=\left(K / r^{2}\right)\left[\partial\left(r^{2} u\right) \partial r\right]=0 \text { at } r=a \\
& \text { I.C } u(r, 0)=\Delta a_{0}(r / a) \quad \text { at } t=0
\end{aligned}
$$

where $D\left(=K / f^{\prime}\right)$ is the diffusion coefficient of the gel; $K$ is the bulk modulus of the polymer network and $f^{\prime}$ is the coefficient of friction between the gel network and the solvent; $a$ is the final radius of the gel sphere in equilibrium with the solvent; $\Delta a_{0}$ is the total increment in the radius of the sphere after swelling; $\sigma_{r r}$ is the normal stress.

As rod-shaped gels having a length about equal to 
the diameter were used in the present study, the radius of the cross section was represented as the radius of an equivalent-volume sphere of the gel.

The exact solution of Eq. (5) can be obtained and the radius of the gel during the course of swelling can be expressed as follows:

$$
\begin{gathered}
\frac{\Delta a(t)}{\Delta a_{0}}=\left(6 / \pi^{2}\right) \Sigma n^{-2} \exp \left(-n^{2} t / t_{s}\right) \\
t_{s}=a^{2} / D
\end{gathered}
$$

Equation (8) can be rewritten in a simple form when $t / t_{s}$ is larger than unity.

$$
\frac{\Delta a(t)}{\Delta a_{0}}=\left(6 / \pi^{2}\right) \exp \left(-t / t_{s}\right)
$$

The swelling time $t$ is scaled by the characteristic time of swelling $t_{s}$.

The ratios $\Delta a(t) / \Delta a_{0}$ are plotted as a function of $t$ in a semilog scale in Figs. 6, 7 and 8 for gels having various constituents as shown in Table 1. A series of curves plotted in these figures show rapid decreases in a certain period just after starting of swelling and then became almost linear. Therefore, the characteristic time $t_{s}$ could be determined from the slope of the straight line calculated by using Eq. (10).

To check experimentally the quantitative validity of Eq. (9), each characteristic time $t_{s}$ obtained from Figs. 6, 7 and 8 was plotted as a function of $a^{2}$, as shown in Fig. 9. A linear relation between them was obtained and is in agreement with the theoretical prediction, $t_{s}=a^{2} / D$. The diffusivities of the gel network were obtained from the slope of each line.

Figure 10 shows typical swelling curves observed experimentally and the calculated ones using the diffusivities of the gel network. The gels which remained shrunken in a solution with somewhat higher than critical concentration of acetone were transferred to another solution with lower concentration of acetone. This time was taken to be zero $(t=0)$. The calculated volume ratios $[d / d(t=0)]^{3}$ agreed fairly well with observed ones.

Figure 11 shows the relationship between the diffusivities, $D$, and the $f$-values of gels having various constituents. The diffusivities correlated well with the $f$-values, as $D=\exp (\alpha f+\beta)$. The values of $\alpha$ and $\beta$ are 0.486 and -13.9 , respectively, under the present conditions. A series of the procedures described above is very useful for analysis of the properties of other gels.

\section{Conclusion}

Poly(acrylamide-co-acrylic acid) gels were synthesized from the monomers acrylamide (Am), acrylic acid (Ac), and $N, N^{\prime}$-methylenebisacrylamide (B). The effects of the relative amounts of these monomers on

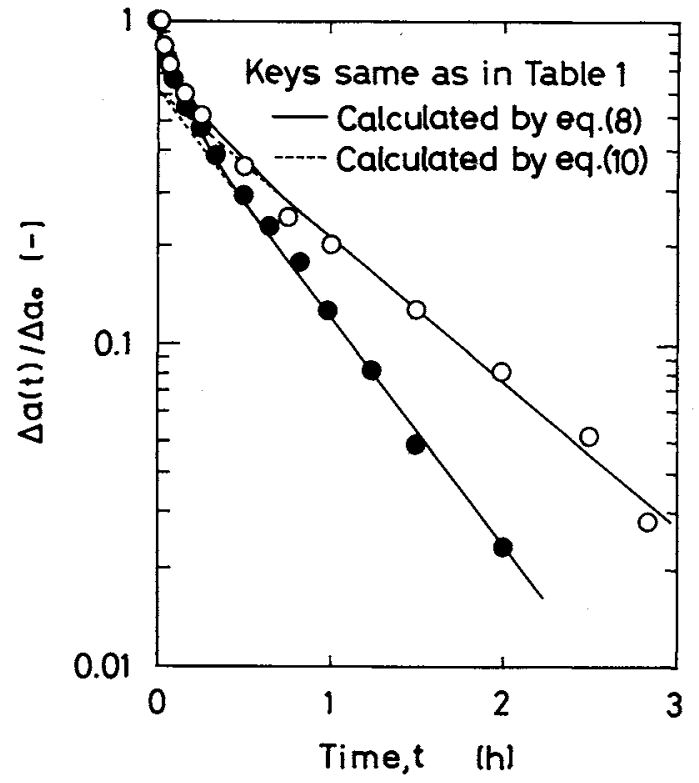

Fig. 6. Time courses of radius of cross section in the gel in which only the concentrations of Ac are varied during swelling. Solid lines are curves calculated using Eq. (8).

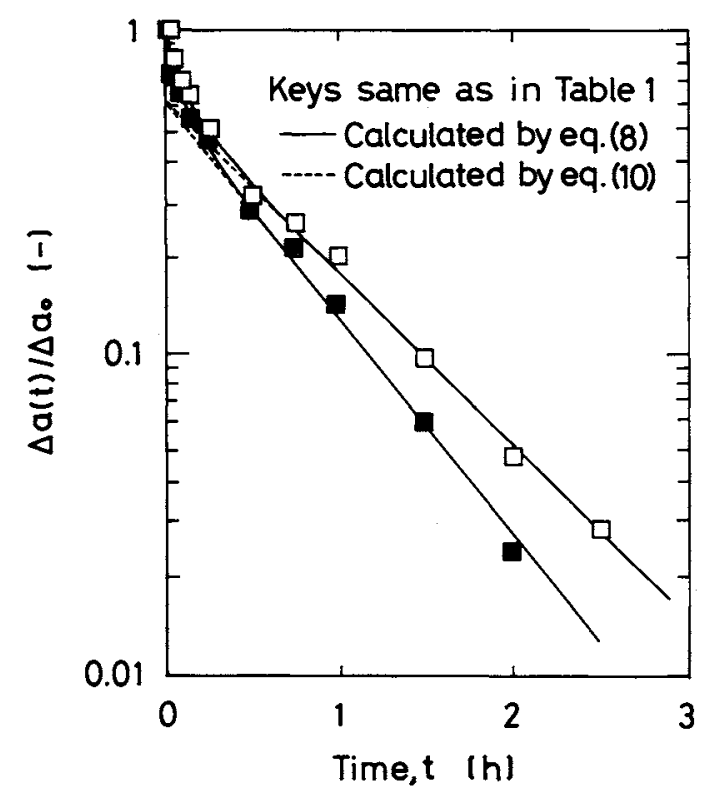

Fig. 7. Time courses of radius of cross section in the gel in which only the concentrations of Am are varied during swelling. Solid lines are curves calculated using Eq. (8).

the phase transition of the copolymer gels were investigated. The macroscopic phenomena of swelling and shrinking by infinitesimal change near a critical concentration of acetone-water mixture were analysed to connect with the $f$-values corresponding to the phase transition.

Gels having relatively high concentrations of Ac showed discontinuous volume changes by a fcator of about 100 , while gels having relatively high concentrations of $\mathrm{Am}$ and/or B showed small volume changes. 


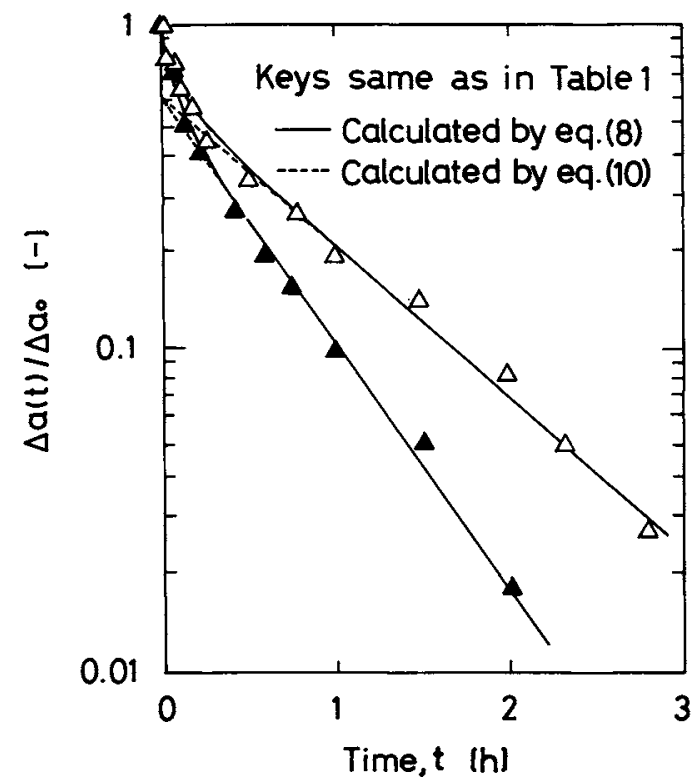

Fig. 8. Time courses of radius of cross section in the gel in which only the concentrations of B are varied, during swelling. Solid lines are curves calculated using Eq. (8)

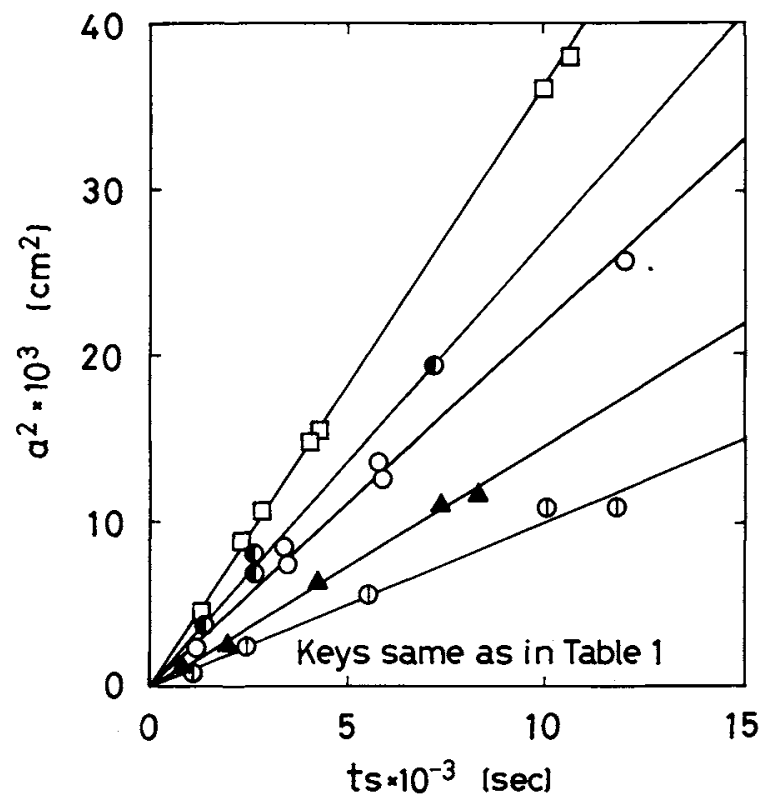

Fig. 9. Characteristic time $t_{s}$ of swelling of poly(acrylamide-co-acrylic acid) gels as a function of the square of final radius $a$.

It was found that the degree of the volume change of the gel can be represented by the $f$-value, mainly corresponding to the amount of ionized acrylic acid, which is defined in the Flory-Huggins theory.

The diffusivity of the gel network was obtained from the concentration swing experiment near the phase transition of gel and the analysis based on Newton's second law and Hooke's law. The diffusivities, $D$, were correlated well with the $f$-values, as follows: $D=\exp (\alpha f+\beta) \alpha=0.486, \beta=-13.9$.

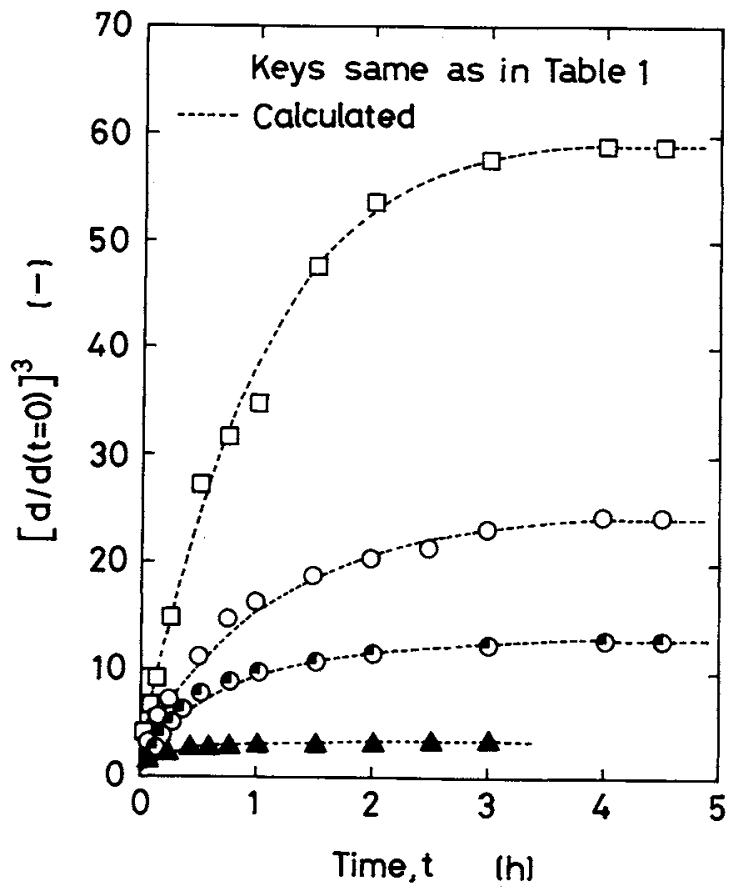

Fig. 10. Comparison of observed gel's swelling with those calculated using the diffusivity of gel network.

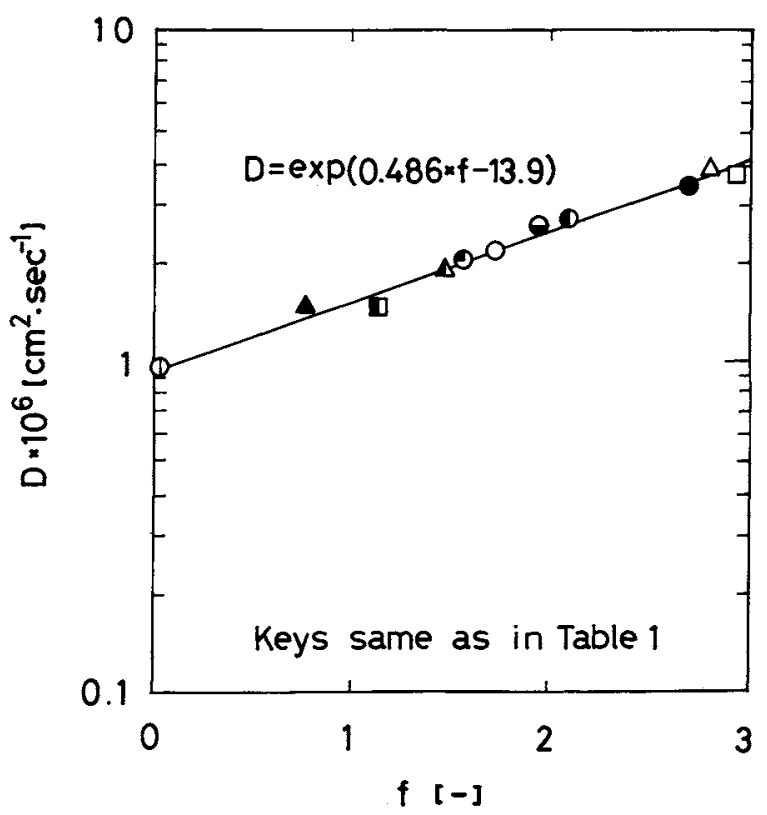

Fig. 11. Relationship between diffisivities of the network in poly(acrylamide-co-acrylic acid) gels and $f$-values.

\section{Acknowledgment}

The authors are grateful to Takayuki Kitano and Shigetaka Yamamoto for their assistance in the experimental work.

\section{Nomenclature}

$\begin{array}{lllr}\mathrm{Ac} & = & \text { molar concentration of acrylic acid } & {[\mathrm{mol} / l]} \\ \mathrm{Am} & = & \text { molar concentration of acrylamide } & {[\mathrm{mol} / l]} \\ a & = & \text { final radius of gel sphere (or the cross-section of } \\ & & \text { rod-shaped gel) in equilibrium with surrounding } \\ & & \text { solvent } & {[\mathrm{cm}, \mathrm{m}]} \\ & = & \text { increment in radius at } t & {[\mathrm{~cm}, \mathrm{~m}]} \\ \Delta a(t) & = & \text { total increment in radius in the entire process }\end{array}$




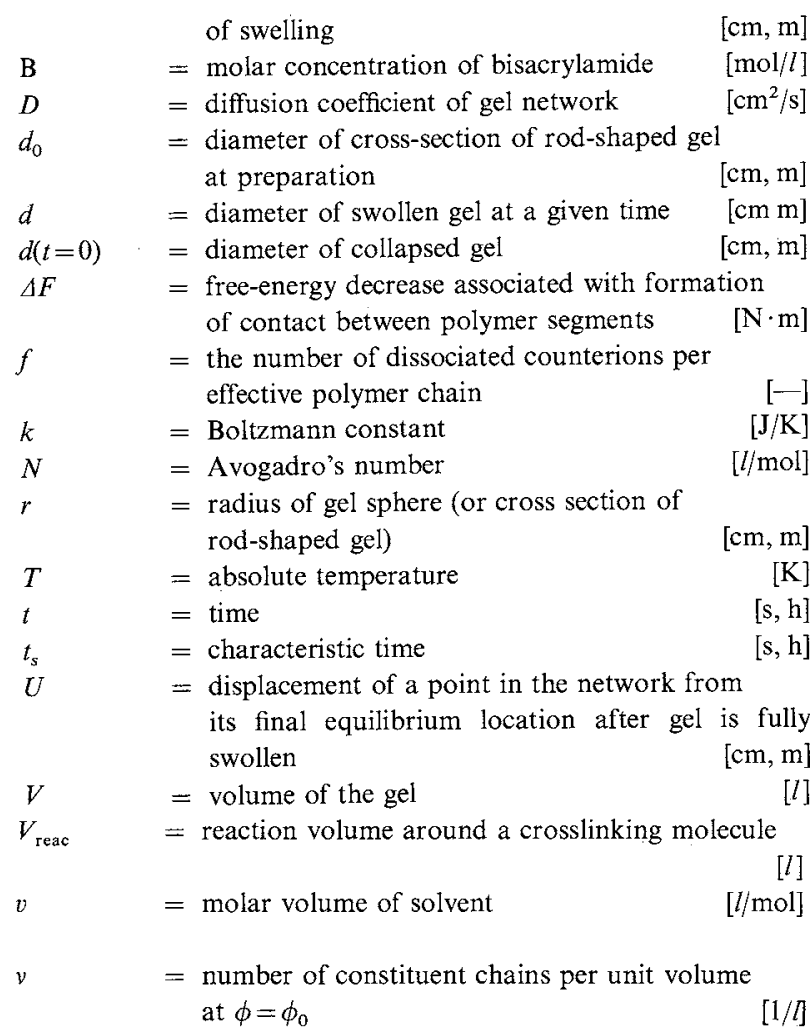

$\begin{array}{llr}\pi & =\text { osmotic pressure of gel } & {\left[\mathrm{N} / \mathrm{m}^{2}\right]} \\ \sigma_{r r} & =\text { normal stress in direction of radius } & {\left[\mathrm{N} / \mathrm{m}^{2}\right]} \\ \tau & =\text { reduced temperature } & {[-]} \\ \phi & =\text { volume fraction of network at equilibrium } & {[-]} \\ \phi_{0} & =\text { volume fraction of network at preparation } & {[-]}\end{array}$

\section{Literature Cited}

1) Cussler, E. L., M. R. Stokar and I. E. Varberg: AIChE J., 30, 578 (1984).

2) Flory, P. J.: "Principles of Polymer Chemistry," Cornell University Press, Ithaca, p. 584 (1953).

3) Hrouz, J., M. Ilavsky, K. Ulbrich and J. Kopecek: Eur. Polym. J., 17, 361 (1981).

4) Ilavsky, M.: Macromolecules, 15, 782 (1982).

5) Ohmine, I. and T. Tanaka: J. Chem. Phys., 11, 5725 (1982).

6) Tanaka, T.: Scientific American, 244, 124 (1981).

7) Tanaka, T., D. J. Fillmore, S.-T. Sun, I. Nishino, G. Swilo and A. Shah: Phys, Rev. Letters, 45, 1636 (1980).

9) Tanaka, T., I. Nishio, S.-T. Sun and S. Ueno-Nishio: Science, 218, 467 (1982).

9) Tanaka, T.: private communication.

10) Tanaka, T. and D. J. Fillmore: J. Chem. Phys, 70, 1214 (1979).

(Presented at the Autumn Meeting of The Society of Chemical Engineers, Japan, at Nagoya, October 18, 1985.) 